\title{
Complete nucleosynthesis calculations for low-mass stars from NuGrid
}

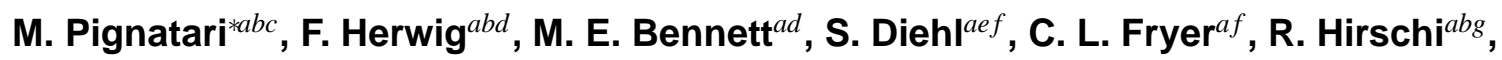 \\ A. Hungerford ${ }^{a f}$, G. Magkotsios ${ }^{a c h}$, G. Rockefeller ${ }^{a f}$, F. X. Timmes ${ }^{a h}$, and P. Young ${ }^{a h}$ \\ ${ }^{a}$ The NuGrid Collaboration \\ ${ }^{b}$ Astrophysics Group, Keele University, ST5 5BG, UK \\ ${ }^{c}$ Joint Institute for Nuclear Astrophysics, University of Notre Dame, IN, 46556, USA \\ ${ }^{d}$ Dept. of Physics \& Astronomy, Victoria, BC, V8W 3P6, Canada \\ ${ }^{e}$ Theoretical Astrophysics Group (T-6), Los Alamos National Laboratory, Los Alamos, NM, \\ 87544, USA \\ ${ }^{f}$ Computational Methods (CCS-2), Los Alamos National Laboratory, Los Alamos, NM, 87544, \\ USA \\ ${ }^{g}$ IPMU, University of Tokyo, Kashiwa, Chiba 277-8582, Japan \\ ${ }^{h}$ School of Earth and Space Exploration, Arizona State University, Tempe, AZ 85287, USA \\ E-mail:marco@astro.keele.ac.uk
}

\begin{abstract}
Many nucleosynthesis and mixing processes of low-mass stars as they evolve from the Main Sequence to the thermal-pulse Asymptotic Giant Branch phase (TP-AGB) are well understood (although of course important physics components, e.g. rotation, magnetic fields, gravity wave mixing, remain poorly known). Nevertheless, in the last years presolar grain measurements with high resolution have presented new puzzling problems and strong constraints on nucleosynthesis processes in stars. The goal of the NuGrid collaboration is to present uniform yields for a large range of masses and metallicities, including low-mass stars and massive stars and their explosions. Here we present the first calculations of stellar evolution and high-resolution, post-processing simulations of an AGB star with an initial mass of $2 \mathrm{M}_{\odot}$ and solar-like metallicity $(\mathrm{Z}=0.01)$, based on the post-processing code $P P N$. In particular, we analyze the formation and evolution of the radiative ${ }^{13} \mathrm{C}$-pocket between the 17 th TP and the 18th TP. The $s$-process nucleosynthesis profile of a sample of heavy isotopes is also discussed, before the next convective TP occurrence.
\end{abstract}

10th Symposium on Nuclei in the Cosmos

July 27 - August 12008

Mackinac Island, Michigan, USA

${ }^{*}$ Speaker. 


\section{Introduction}

When $\mathrm{He}$-burning is exhausted in the core, low mass stars $\left(1.5-3 \mathrm{M}_{\odot}\right)$ evolve along the AGB. Late on the AGB, recurrent Thermal Pulses affect shell He-burning history ([1]). After TPs (time scale in the order of few hundreds years), the third dredge-up events (TDU) mix He shell material in the envelope, and fresh protons down in the $\mathrm{He}$-intershell. $\mathrm{A}^{13} \mathrm{C}$-pocket is formed in the radiative $\mathrm{He}$ intershell phase, where the ${ }^{13} \mathrm{C}(\alpha, \mathrm{n}){ }^{16} \mathrm{O}$ neutron source becomes efficient activating slow neutron capture process ( $\mathrm{s}-$ process, [2]). A marginal contribution is also given by the partial activation of the ${ }^{22} \mathrm{Ne}(\alpha, \mathrm{n})^{25} \mathrm{Mg}$ at the bottom of the He intershell (e.g., [3]).

As a result of the TDU enriching the AGB envelope with $s$-process rich material, AGB stars provide most of the $s$ elements beyond Sr observed in the Solar System. In particular, the "main component" between $\mathrm{Sr}$ and $\mathrm{Pb}$ is produced by solar-like AGB stars, while the "strong component" explaining half of the solar ${ }^{208} \mathrm{~Pb}$ is produced by low metallicity AGB stars ([4], and references therein). Carbon is also dredged-up with s elements in the envelope, and the AGB star may become a C-rich star $(\mathrm{C}(\mathrm{N})$ star), meaning that carbon is more abundant than oxygen in the envelope.

Spectroscopic observations and composition measurements in presolar grains formed in AGB stars confirm this scenario ([5],[6],respectively), and provide important insight to study and understand those stars in more details. In particular, presolar grains carry the isotopic and chemical signature of their parent stars (e.g., [7],[6],[8]), providing a powerful tool to test and constrain stellar models and nuclear physics inputs. The NuGrid project (see Herwig et al. in this volume) has the goal to generate uniform yields for a large range of masses and metallicities including low-mass stars, and to constrain them with observations. In this proceeding we present our first calculations of stellar evolution and high-resolution, post-processing simulations of a $2 \mathrm{M}_{\odot} \mathrm{Z}=0.01$ AGB star, based on the post-processing code $P P N$.

\section{Post-processing calculations}

The main input parameters for the post-processing calculations are given by a $2 \mathrm{M}_{\odot}$ and $\mathrm{Z}=$ 0.01 star (EVOL Code, [9], model ET2). In the stellar model the overshoot parameter $f$ has been semi-empirically determined, where the $f$ applied at the base of the convective TP in the He shell $(f=0.008)$ has been constrained to explain the $\mathrm{He} / \mathrm{C} / \mathrm{O}$ ratio observed in $\mathrm{H}$ deficient post-AGB stars of type PG1159 and in WC central stars of planetary nebulae ([10]), and the $f$ at the bottom of the envelope $(f=0.128)$ is calibrated to reproduce the mass of the ${ }^{13} \mathrm{C}$-pocket to reproduce the observed overabundance of $s$-process elements [11]. Notice that, according to [9] (Fig. 2 in that paper), the present model is in agreement with the bolometric magnitude $\left(\mathrm{M}_{\mathrm{bol}}\right)$ observed for TP-AGB stars (e.g. [12]). PPN is described in Herwig et al. (this vol.) and includes dynamically all species from $\mathrm{H}$ to $\mathrm{Bi}$. Concerning the simulations shown in this proceeding, [13] (NACRE compilation) is used for the main charged particle reactions, [14] (Kadonis compilation) for neutron capture reactions and [15] for unstable isotopes not included in Kadonis. [16] and [17] are used for stellar $\beta$-decay rates of light unstable isotopes, and [18] for $\beta$-decay rates of heavy unstable isotopes (the terrestrial rates are used if not available in the previous references). 

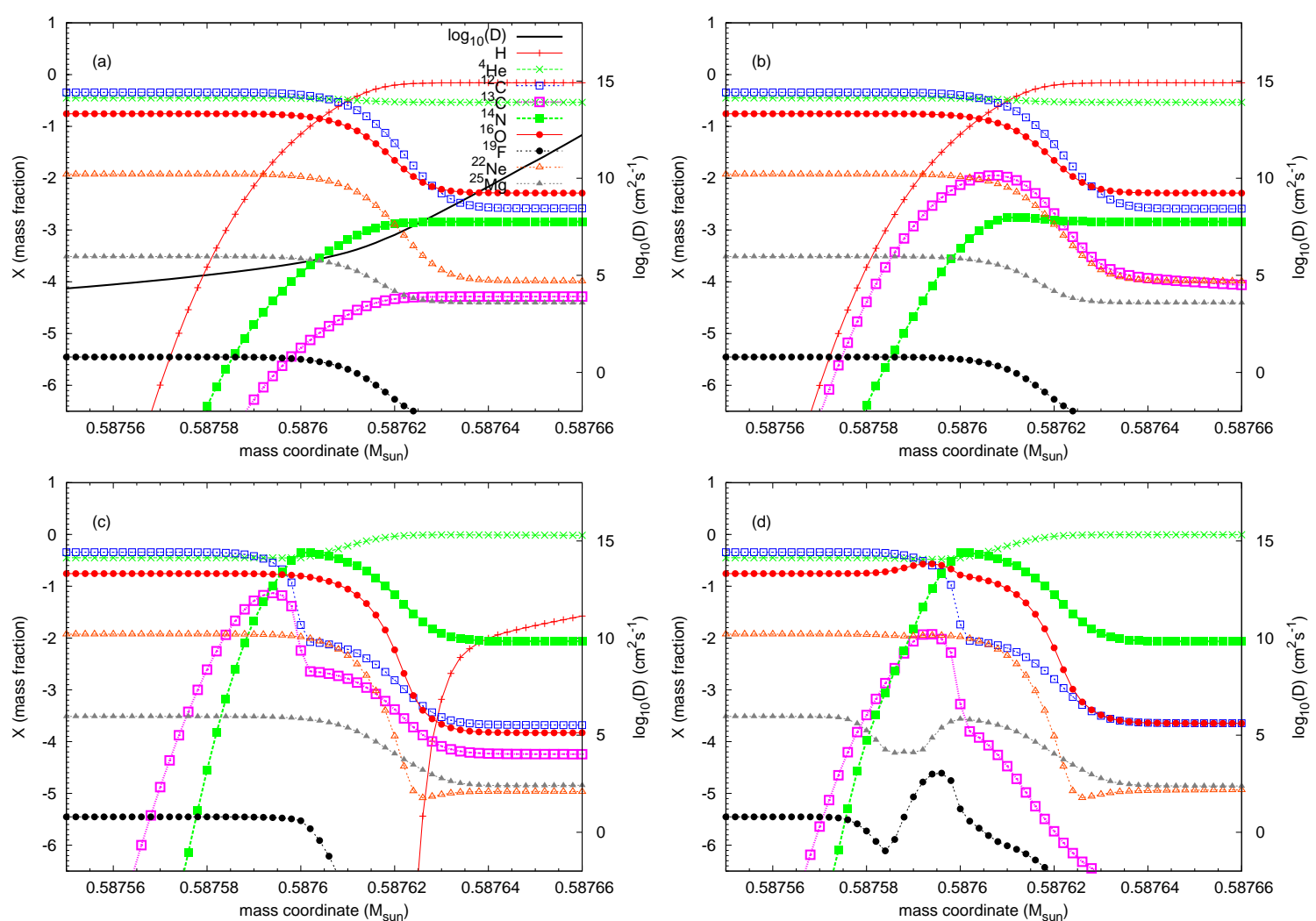

Figure 1: Panel (a,b,c,d): Formation and evolution of the ${ }^{13} \mathrm{C}$-pocket after the 17 th TP for the $2 \mathrm{M}_{\odot} \mathrm{Z}=0.01$ star. The profile for the mixing coefficient D (it is different from zero only in Panel (a)) and for a sample of light isotopes is provided.

In Fig. 1 we show the ${ }^{13} \mathrm{C}$-pocket formation by overshooting (e.g. [9],[11]) and its evolution between the 17th TP and the 18th TP (interpulse phase of about $70000 \mathrm{yr}$ ). In Panel (a) during the TDU protons and envelope material are mixed down in the $\mathrm{He}$ intershell, before the re-activation of the $\mathrm{H}$ shell. In Panel (b) the abundant ${ }^{12} \mathrm{C}$ is efficiently capturing protons producing ${ }^{13} \mathrm{C}$ in radiative conditions via the nucleosynthesis channel ${ }^{12} \mathrm{C}(\mathrm{p}, \gamma){ }^{13} \mathrm{~N}\left(\beta^{+}\right){ }^{13} \mathrm{C}$ (e.g., [19]). At the ${ }^{13} \mathrm{C}$ abundance peak, also ${ }^{14} \mathrm{~N}$ starts to be produced by the proton capture channel ${ }^{13} \mathrm{C}(\mathrm{p}, \gamma){ }^{14} \mathrm{~N}$. In Panel (c) the ${ }^{13} \mathrm{C}$-pocket final shape is shown, since protons are fully consumed. The pocket size is $1-2 \times 10^{-5} \mathrm{M}_{\odot}$. Moving outward, a prominent ${ }^{14} \mathrm{~N}$-pocket is formed just after the ${ }^{13} \mathrm{C}$-pocket, as expected. After about 40000 years from the previous TP, the temperature in the radiative ${ }^{13} \mathrm{C}$-pocket is high enough to efficiently activate the ${ }^{13} \mathrm{C}(\alpha, n){ }^{16} \mathrm{O}$ reaction, 
producing neutrons for the $s$ process. In Panel (d) we show the ${ }^{13} \mathrm{C}$-pocket region once ${ }^{13} \mathrm{C}$ has been burnt and the $s$ process is not anymore efficient. ${ }^{25} \mathrm{Mg}$ is the main neutron poison in the ${ }^{13} \mathrm{C}$ pocket, and it has been partially depleted by the neutron flux. On the other hand, in the ${ }^{14} \mathrm{~N}$-pocket ${ }^{14} \mathrm{~N}(n, p){ }^{14} \mathrm{C}$ is the main neutron poison as it is well known, and the neutron capture efficiency of ${ }^{25} \mathrm{Mg}$ (and of the main $s$-process seed ${ }^{56} \mathrm{Fe}$ ) quickly decreases with increasing the ${ }^{14} \mathrm{~N}$ abundance. In the ${ }^{13} \mathrm{C}$-pocket ashes the ${ }^{19} \mathrm{~F}$ abundance profile mimics the ${ }^{13} \mathrm{C}$ profile. In particular, ${ }^{19} \mathrm{~F}$ is depleted by neutron capture and by $\alpha$ capture if ${ }^{13} \mathrm{C}$ is more abundant than ${ }^{14} \mathrm{~N}$ or, in case ${ }^{14} \mathrm{~N}$ is more abundant than ${ }^{13} \mathrm{C},{ }^{19} \mathrm{~F}$ is produced starting from ${ }^{14} \mathrm{~N}$ [20]. Finally, in Fig. 2, we report the final overabundance profile in the ${ }^{13} \mathrm{C}$-pocket ashes for a sample of isotopes at the $\mathrm{Sr}$ neutron magic peak $\left({ }^{86,87,88} \mathrm{Sr}\right)$, at the Ba neutron magic peak $\left({ }^{136,138} \mathrm{Ba},{ }^{139} \mathrm{La}\right)$ and at the $\mathrm{Pb}$ neutron magic peak $\left({ }^{206,208} \mathrm{Ba}\right)$. The $l s$ peak species (e.g. [5]) show a maximum of overproduction of about $5 \times 10^{3}$, while the $h s$ peak and the $\mathrm{Pb}$ peak species show an overproduction of about few $10^{4}$. Sr isotopes show a double peak in coincidence of the ${ }^{56} \mathrm{Fe}$ depletion tails. At $0.58758 \mathrm{M}_{\odot}$ the ${ }^{13} \mathrm{C}$ abundance is rapidly decreasing and as a consequence a lower amount of neutrons are produced. On the other hand, at $0.587595 \mathrm{M}_{\odot}$ the poisoning effect of ${ }^{14} \mathrm{~N}$ is increasing, until the ${ }^{56} \mathrm{Fe}$ neutron capture efficiency is negligible. The $\mathrm{Ba}$ peak and the $\mathrm{Pb}$ peak are more produced in the center of the ${ }^{13} \mathrm{C}$ pocket, where lighter Sr peak elements are feeding $s$ nucleosynthesis of heavier elements. The next convective TP will mix the $s$-process rich pocket in all the He intershell, which will be partially dredged up in the envelope by the next TDU event.

The analysis presented in this work shows part of the capabilities of PPN applied to AGB nucleosynthesis. At present, we may calculate isotopic and element abundances from $\mathrm{H}$ to $\mathrm{Bi}$ at any position and at any time in a complete stellar track. Furthermore, in the nuclear network every reaction rate may be automatically chosen between different nuclear sources, or a multiplication factor can be applied or the reaction may be not considered. This opens up possibilities to systematically take into account the effect of nuclear uncertainties in our nucleosynthesis calculations.

\section{Acknowledgments}

M.P. acknowledges support through NSF grants PHY 02-16783 (JINA). M.P. and F.H. were supported by a Marie Curie International Reintegration Grant MIRG-CT-2006-046520 within the European FP6.

\section{References}

[1] M. Schwarzschild and R. Härm, Thermal Instability in Non-Degenerate Stars., ApJ 142 (Oct., 1965) 855.

[2] E. M. Burbidge, G. R. Burbidge, W. A. Fowler, and F. Hoyle, Synthesis of the Elements in Stars, Reviews of Modern Physics 29 (1957) 547-650.

[3] R. Gallino, C. Arlandini, M. Busso, M. Lugaro, C. Travaglio, O. Straniero, A. Chieffi, and M. Limongi, Evolution and Nucleosynthesis in Low-Mass Asymptotic Giant Branch Stars. II. Neutron Capture and the s-Process, ApJ 497 (Apr., 1998) 388-+.

[4] C. Arlandini, F. Käppeler, K. Wisshak, R. Gallino, M. Lugaro, M. Busso, and O. Straniero, Neutron Capture in Low-Mass Asymptotic Giant Branch Stars: Cross Sections and Abundance Signatures, ApJ 525 (Nov., 1999) 886-900, [arXiv: astro-ph/9906266]. 
[5] M. Busso, R. Gallino, D. L. Lambert, C. Travaglio, and V. V. Smith, Nucleosynthesis and Mixing on the Asymptotic Giant Branch. III. Predicted and Observed s-Process Abundances, ApJ 557 (Aug., 2001) 802-821, [arXiv: astro-ph/0104424].

[6] M. Lugaro, A. M. Davis, R. Gallino, M. J. Pellin, O. Straniero, and F. Käppeler, Isotopic Compositions of Strontium, Zirconium, Molybdenum, and Barium in Single Presolar SiC Grains and Asymptotic Giant Branch Stars, ApJ 593 (Aug., 2003) 486-508.

[7] E. Zinner, Stellar Nucleosynthesis and the Isotopic Composition of Presolar Grains from Primitive Meteorites, Annual Review of Earth and Planetary Sciences 26 (1998) 147-188.

[8] J. G. Barzyk, M. R. Savina, A. M. Davis, R. Gallino, M. J. Pellin, R. S. Lewis, S. Amari, and R. N. Clayton, Multi-element isotopic analysis of single presolar SiC grains, New Astronomy Review 50 (Oct., 2006) 587-590.

[9] F. Herwig and S. M. Austin, Nuclear Reaction Rates and Carbon Star Formation, ApJ 613 (Sep., 2004) L73-L76, [arXiv:astro-ph/0 408394 ].

[10] K. Werner and F. Herwig, The Elemental Abundances in Bare Planetary Nebula Central Stars and the Shell Burning in AGB Stars, PASP 118 (Feb., 2006) 183-204, [arXiv: astro-ph/0 512320 ].

[11] M. Lugaro, F. Herwig, J. C. Lattanzio, R. Gallino, and O. Straniero, s-Process Nucleosynthesis in Asymptotic Giant Branch Stars: A Test for Stellar Evolution, ApJ 586 (Apr., 2003) 1305-1319, [arXiv:astro-ph/0212364].

[12] R. Guandalini, M. Busso, S. Ciprini, G. Silvestro, and P. Persi, Infrared photometry and evolution of mass-losing AGB stars. I. Carbon stars revisited, A\&A 445 (Jan., 2006) 1069-1080, [arXiv:astro-ph/0509739].

[13] C. Angulo, M. Arnould, M. Rayet, P. Descouvemont, D. Baye, C. Leclercq-Willain, A. Coc, S. Barhoumi, P. Aguer, C. Rolfs, R. Kunz, J. W. Hammer, A. Mayer, T. Paradellis, S. Kossionides, C. Chronidou, K. Spyrou, S. Degl'Innocenti, G. Fiorentini, B. Ricci, S. Zavatarelli, C. Providencia, H. Wolters, J. Soares, C. Grama, J. Rahighi, A. Shotter, and M. Lamehi Rachti, A compilation of charged-particle induced thermonuclear reaction rates., Nuclear Physics A 656 (Aug., 1999) 3-183.

[14] I. Dillmann, R. Plag, M. Heil, F. Käppeler, and T. Rauscher, Present status of the KADoNiS database, in International Symposium on Nuclear Astrophysics - Nuclei in the Cosmos, 2006.

[15] T. Rauscher and F.-K. Thielemann, Astrophysical Reaction Rates From Statistical Model Calculations, Atomic Data and Nuclear Data Tables 75 (May, 2000) 1-2, [arXiv: astro-ph/ 0004059 ].

[16] T. Oda, M. Hino, K. Muto, M. Takahara, and K. Sato, Rate Tables for the Weak Processes of sd-Shell Nuclei in Stellar Matter, Atomic Data and Nuclear Data Tables 56 (1994) 231-+.

[17] G. M. Fuller, W. A. Fowler, and M. J. Newman, Stellar weak interaction rates for intermediate-mass nuclei. IV - Interpolation procedures for rapidly varying lepton capture rates using effective log (ft)-values, ApJ 293 (Jun., 1985) 1-16.

[18] S. Goriely, Uncertainties in the solar system r-abundance distribution, A\&A 342 (Feb., 1999) 881-891.

[19] O. Straniero, R. Gallino, M. Busso, A. Chieffi, C. M. Raiteri, M. Limongi, and M. Salaris, Radiative C-13 burning in asymptotic giant branch stars and s-processing, ApJ 440 (Feb., 1995) L85-L87.

[20] A. Jorissen, V. V. Smith, and D. L. Lambert, Fluorine in red giant stars - Evidence for nucleosynthesis, A\&A 261 (Jul., 1992) 164-187. 